\title{
Induced Chirality in Halide Perovskite Clusters through Surface Chemistry
}

Aaron Forde ${ }^{1-3^{*}}$, Dibyajyoti Ghosh ${ }^{2-4}$, Dmitri Kilin ${ }^{5}$, Amanda C. Evans ${ }^{6}$, Sergei Tretiak ${ }^{2}$, Amanda J Neukirch $^{2 *}$

${ }^{1}$ Department of Materials Science and Nanotechnology, North Dakota State University, Fargo, North Dakota 58102, United States

${ }^{2}$ Theoretical Physics and Chemistry of Materials, Los Alamos National Laboratory, Los Alamos, New Mexico 87545, United States

${ }^{3}$ Center for Nonlinear Studies, Los Alamos National Laboratory, Los Alamos National Laboratory, Los Alamos, New Mexico 87545, United States

${ }^{4}$ Department of Materials Science and Engineering and Department of Chemistry, Indian Institute of Technology, Delhi,, Hauz Khas, New Delhi 110016, India

${ }^{5}$ Department of Chemistry and Biochemistry, North Dakota State University, Fargo, North Dakota 58102, United States

${ }^{6}$ Bioscience Division, Los Alamos National Laboratory, Los Alamos, New Mexico 87545, United States

*Corresponding Authors: Aaron Forde (aforde@lanl.gov) and Amanda Neukirch (ajneukirch@lanl.gov)

\section{Methods}

Theory:

Ground-state geometry and electronic structure are found from the self-consistent DFT equations which minimizes total energies. To determine the strength of interaction between a chiral molecule and the cluster surface we compute binding energies, eq. 1, where $E_{\text {binding }}>0$ indicates unfavorable binding and $E_{\text {binding }}<0$ indicates favorable binding.

$$
E_{\text {binding }}=E(\text { cluster }+ \text { molecule })-[E(\text { molecule })+E(\text { cluster })]
$$

To quantify the impact of the change in ionic coordinates when molecules bind to the cluster surface we compute partial radial distribution functions (pRDF), eq 2, and partial angular distribution functions (pADF), eq 3,

$$
g(r)=\frac{1}{4 \pi r^{2}} \sum_{I J} \delta\left(r-\left|\vec{R}_{I}-\vec{R}_{J}\right|\right)
$$




$$
g(\theta)=\sum_{I J} \delta\left(\theta-\cos ^{-1}\left(\frac{\vec{R}_{I} \vec{R}_{J}}{\left|\vec{R}_{I}\right|\left|\vec{R}_{J}\right|}\right)\right)
$$

where $I, J$ refer to specific set of atomic species. Note that for pADFs the scan over nuclear coordinates is restricted to the $1^{\text {st }}$ coordination peak of the pRDFs so that only nearest neighbor coordination's are considered.

Excited-electronic states are found using time-dependent density functional theory (TDDFT) by solving the Casida equations. Briefly, the solutions of the Casida equation produce eigenvectors $X_{n}$ and $Y_{n}$, which represent transition densities, with eigenvalues $\Omega_{n}$ which represent the transition frequencies. For chiroptical activity the optical transitions of interest are the electronic transition dipole, eq 4 and magnetic transition dipole, eq 5 . In a single particle basis the transition dipoles are represented as

$$
\begin{aligned}
& \vec{\mu}_{i j}^{e l e c}=-e\left\langle\psi_{i}|\vec{r}| \psi_{j}\right\rangle \\
& \vec{\mu}_{i j}^{m a g}=-\frac{e}{2 m c}\left\langle\psi_{i}|\vec{r} \times \vec{p}| \psi_{j}\right\rangle
\end{aligned}
$$

where $\left|\psi_{j}\right\rangle$ and $\left.\psi_{i}\right\rangle$ are single particle Kohn-Sham eigenstates. The excitonic dipole moments from TDDFT are found from expectation values of the transition densities ${ }^{1}$

$$
\begin{array}{r}
\mu_{0 k}^{e l e c}=\sum_{i j} \vec{\mu}_{i j}^{e l e c}\left(X_{i j, k}+Y_{i j, k}\right) \\
\mu_{0 k}^{m a g}=\sum_{i j} \vec{\mu}_{i j}^{\text {mag }}\left(X_{i j, k}-Y_{i j, k}\right)
\end{array}
$$

From the excitonic transition dipoles the corresponding oscillator strength, eq 8 , and rotatory strength, eq 9, for an electronic transition of frequency $\Omega_{n}$ can be determined where they describe the probability of absorbing linearly polarized light and circularly polarized light

$$
f_{0 k} \propto\left|\vec{\mu}_{0 k}^{\text {elec }}\right|^{2}
$$




$$
R_{0 k} \propto \operatorname{Im}\left(\left|\vec{\mu}_{0 k}^{e l e c} \cdot \vec{\mu}_{k 0}^{\operatorname{mag}}\right|\right)
$$

The total absorption and circular dichroism spectra can then be generated with equation 10 and 11

$$
\begin{array}{r}
A\left(\Omega_{k}\right)=\sum_{k} f_{0 k} \delta\left(\hbar \Omega-\hbar \Omega_{k}\right) \\
C D\left(\Omega_{k}\right)=\sum_{k} R_{0 k} \delta\left(\hbar \Omega-\hbar \Omega_{k}\right)
\end{array}
$$

where the delta function $\delta$ is broadened as a gaussian distribution with a broadening parameter $10 \mathrm{meV}$.

To quantify the charge-transfer character of an excitation ${ }^{4}$ we partition the model into fragments of the perovskite cluster and the DACH molecule on the surface and compute fraction of charge density on each fragment

$$
P_{\text {cluster }, n}=\frac{1}{\sum_{\text {frag }}^{\text {frag cluster, molecule }} C_{\text {frag, } n}^{*} C_{\text {frag }, n}} \sum_{\text {frag }}=\text { cluster } C_{\text {frag, },}^{*} C_{\text {frag }, n}
$$

where $P_{\text {cluster }, n}$ is the fraction of charge density assigned to the cluster for molecular orbital $n$ and $C_{i, n}^{*}$ is the expansion coefficient for atomic orbital $i$ that composes $\mathrm{MO} n$. The charge

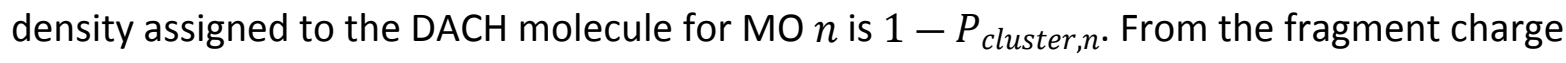
densities we compute charge transfer excitation metric $D_{k}$ which weights each contribution by the transition density coefficient

$$
D_{k}=\sum_{i \geq L U, j \leq H O} X_{k, i j}^{2}\left(P_{i}-P_{j}\right)
$$

where $P_{i}$ and $P_{j}$ refer to fragment partial density of states of the perovskite cluster in the conduction band MOs $i$ and valence band MOs $j$. Positive values indicate charge transfer from the molecule to the cluster and negative values indicate charge transfer from the cluster to the molecule. 
To compare the relative intensities of the linear and polarized absorption we compute

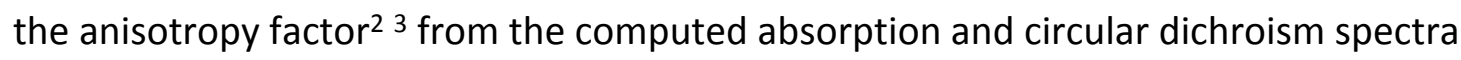

$$
g=\frac{\Delta A}{A}=2 \frac{A^{L H P}-A^{R H P}}{A^{L H P}+A^{R H P}}=\frac{1 \quad C D\left(\Omega_{k}\right)}{32980 A\left(\Omega_{k}\right)}
$$

\section{Computational Details}

Atomistic Models: Enantiomeric structures for the organic molecules studied here where found from PubChem database. The structure for the inorganic cluster was carved from bulk. To give the cluster mirror symmetry the chemical composition of the structure deviates from stoichiometry to give $\mathrm{Cs}_{19} \mathrm{~Pb}_{8} \mathrm{Cl}_{36}$ composition with a $\mathrm{Cs}-\mathrm{Cl}$ terminated surface.

Basis Set and Functionals: All calculations were done using Gaussian $16^{5}$. Ground state optimization was done using B3LYP functional with the organic molecules having the 6$311++\mathrm{g} * *$ basis set. The perovskite clusters were optimized using pseudopotential LANL2DZ basis with B3LYP functional. For the joint systems when $1 \mathrm{X}, 2 \mathrm{X}$ DACH was placed on the surface of the perovskite cluster the model was optimized using the B3LYP functional with LANL2DZ basis set.

For TDDFT calculations CAM-B3LYP functional was used for all calculations with varying basis sets for the $1 \mathrm{X}, 2 \mathrm{X}$ DACH molecules, inorganic cluster, and the combined systems. Basis sets used are LANL2DZ for the perovskite clusters, $6-311++g * *$ for the organic molecules, and a mixed basis set for the combined systems where the perovskite clusters are treated with LANL2DZ and the DACH molecules are treated with 6-311++g**. Computed rotatory strengths 
are sensitive to the basis set size. Comparison between the length and velocity gauge rotatory strengths gives an indication of how complete the basis-set is. Comparison of velocity and length gauges at the $6-311++g * *$ level give nearly equivalent results.

\section{Structural Characterization}

(a)

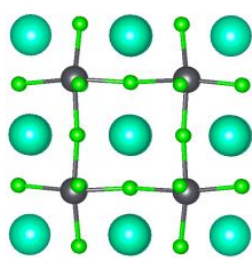

(b)

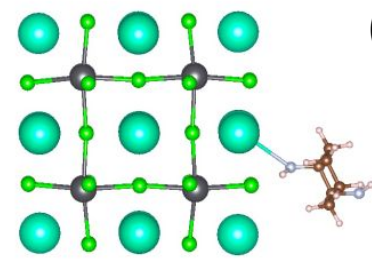

(c)

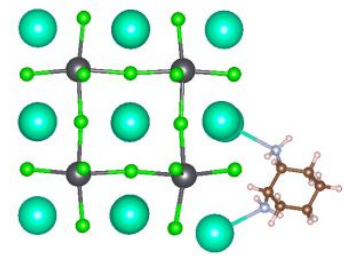

(d)

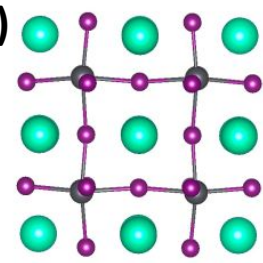

(e)

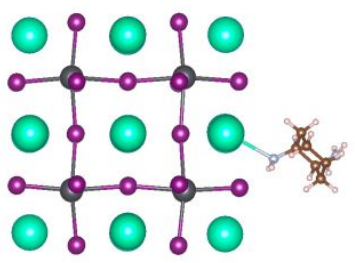

(f)

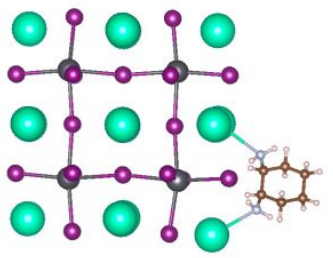

Figure S1: Geometry optimized structures for (a) bare $\mathrm{CsPbCl} 3$ cluster, (b) $\mathrm{CsPbCl} 3$ cluster with $1 \mathrm{R}, 2 \mathrm{R}$ DACH binding in monodentate and (c) bridging configuration. (d) Bare CsPbl3 cluster, (e) $\mathrm{CsPbCl} 3$ cluster with $1 \mathrm{R}, 2 \mathrm{R}$ DACH binding in monodentate and ( $\mathrm{f}$ ) bridging configuration. It is observed that in the monodentate configuration the structure of cluster is virtually unaltered while in the bridging configuration the surface $\mathrm{Cs}$ that coordinate to the $\mathrm{DACH}$ molecule are distorted with respect to the bare cluster. 

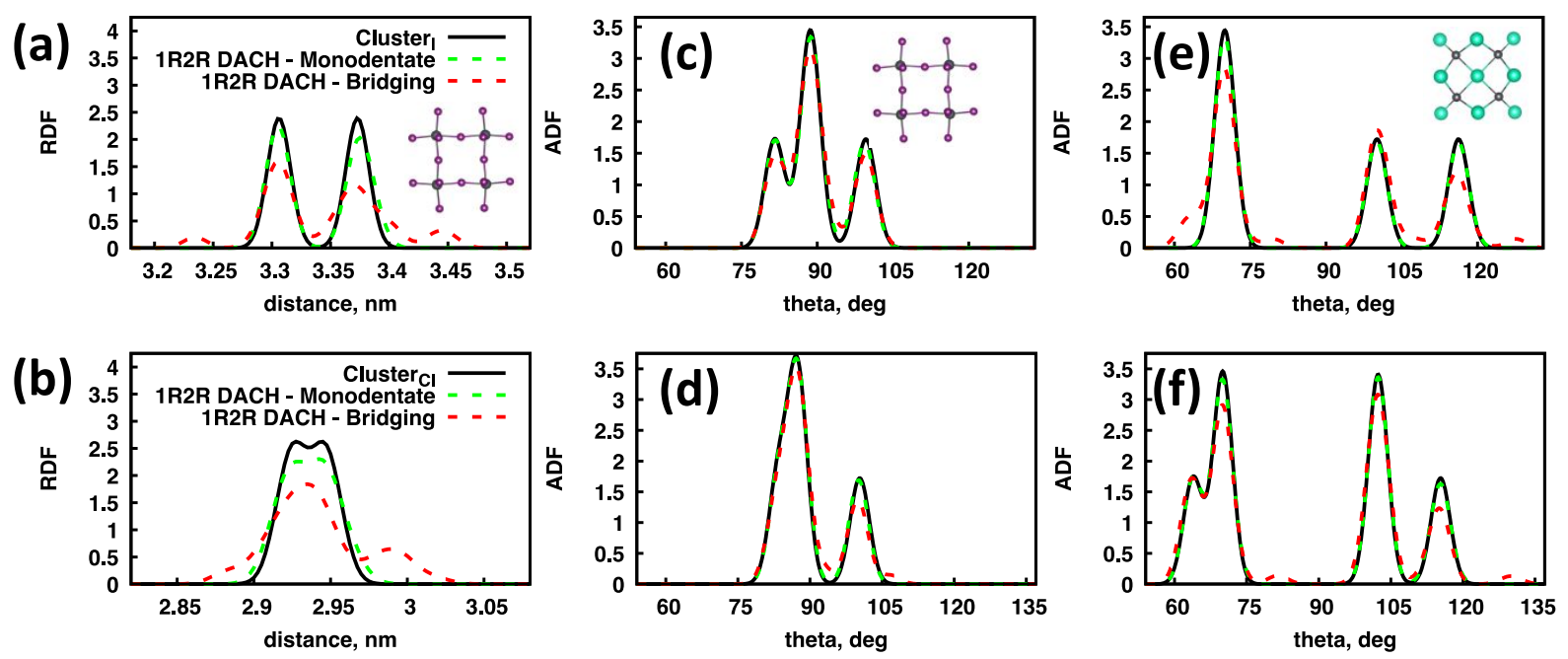

Figure S2: Partial radial distribution functions for (a)-(b) $\mathrm{Pb}-\mathrm{X}(\mathrm{X}=\mathrm{Cl}, \mathrm{I})$ nearest neighbor coordination distances and angular distribution functions for (c)-(d) X-Pb-X and (e)-(f) Cs-Pb-Cs nearest neighbor angles, showing the changes in coordination distances and bonding angles for the bare cluster and when a 1R,2R DACH molecule binds to the surface. It is observed that when the DACH molecule binds in the monodentate configuration there is a slight broadening of coordination distances and angles while the bridging configuration provides greater distortions. 
Spectra of Distorted Cluster + 1X,2X DACH
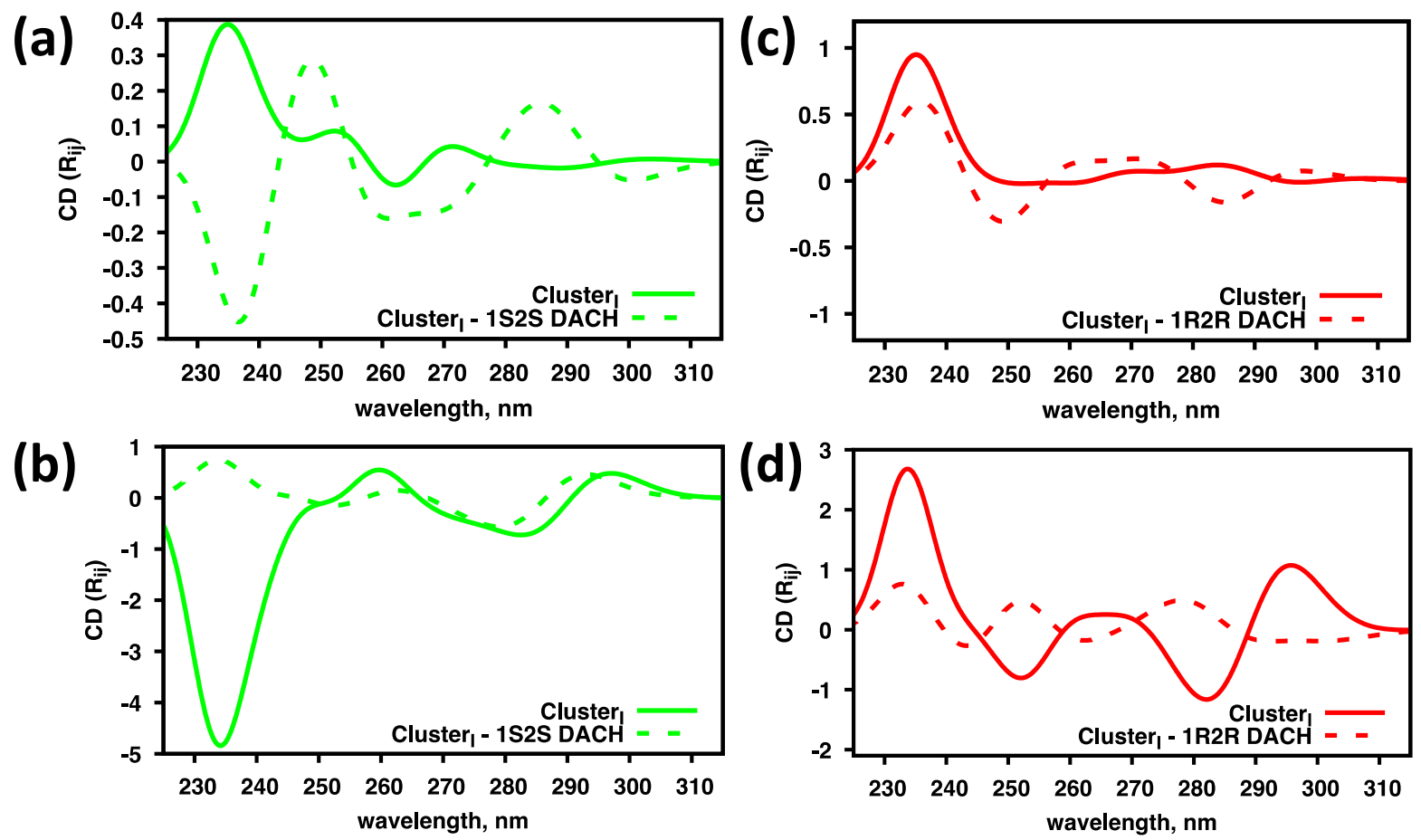

Figure S3: Comparison of the $\mathrm{CsPb}_{3}$ distorted cluster $\mathrm{CD}$ spectra, without $\mathrm{DACH}$ enantiomer bound to the surface, with the combined system for (a) monodentate and (b) bridging configuration for $1 \mathrm{~S}, 2 \mathrm{~S} \mathrm{DACH}$ and (c) monodentate and (d) bridging configuration for $1 \mathrm{R}, 2 \mathrm{R}$ DACH. 

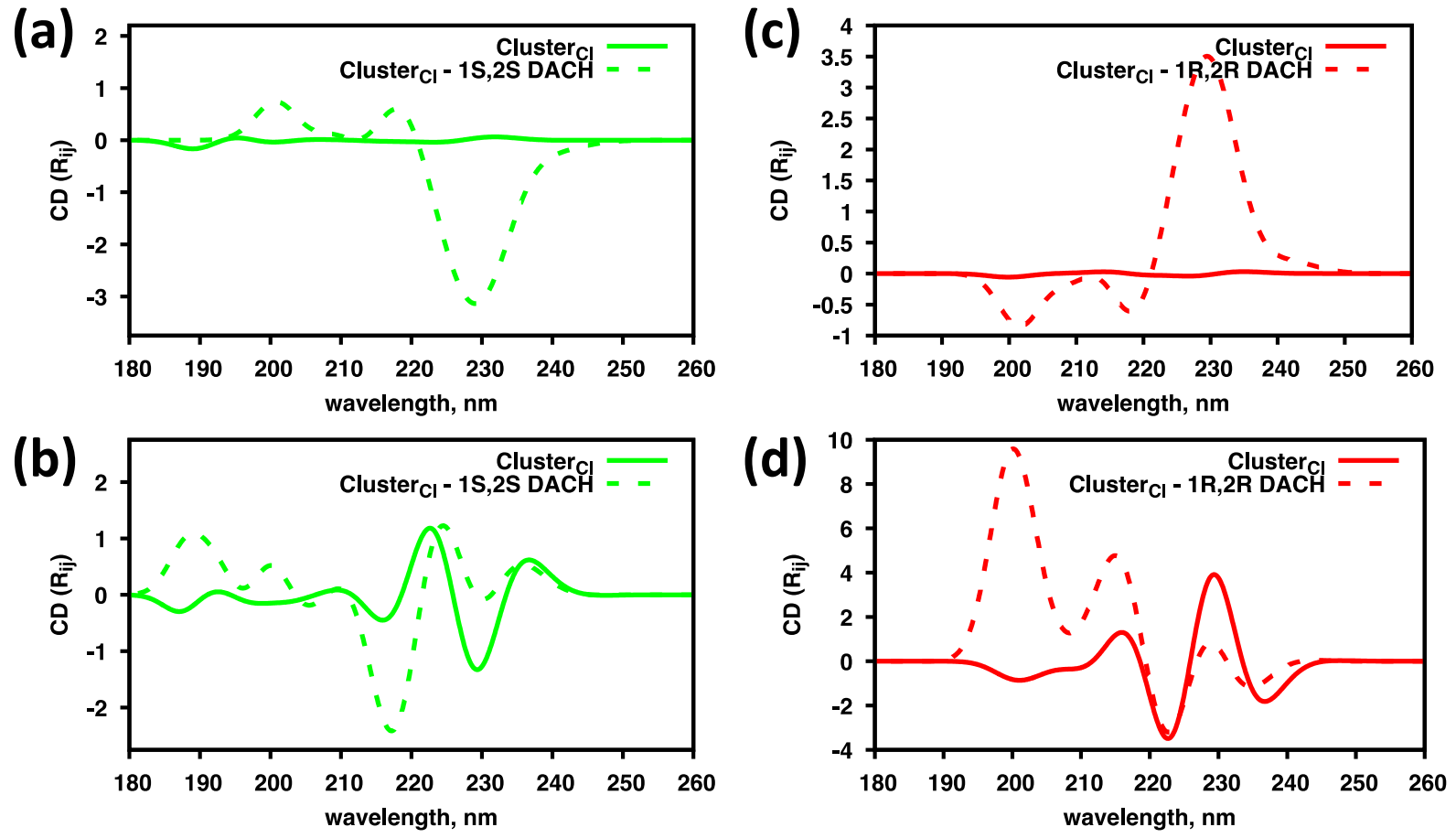

Figure S4: Comparison of the $\mathrm{CsPbCl}_{3}$ distorted cluster $\mathrm{CD}$ spectra, without $\mathrm{DACH}$ enantiomer bound to the surface, with the combined system for (a) monodentate and (b) bridging configuration for $1 \mathrm{~S}, 2 \mathrm{~S} \mathrm{DACH}$ and (c) monodentate and (d) bridging configuration for $1 \mathrm{R}, 2 \mathrm{R}$ DACH. 

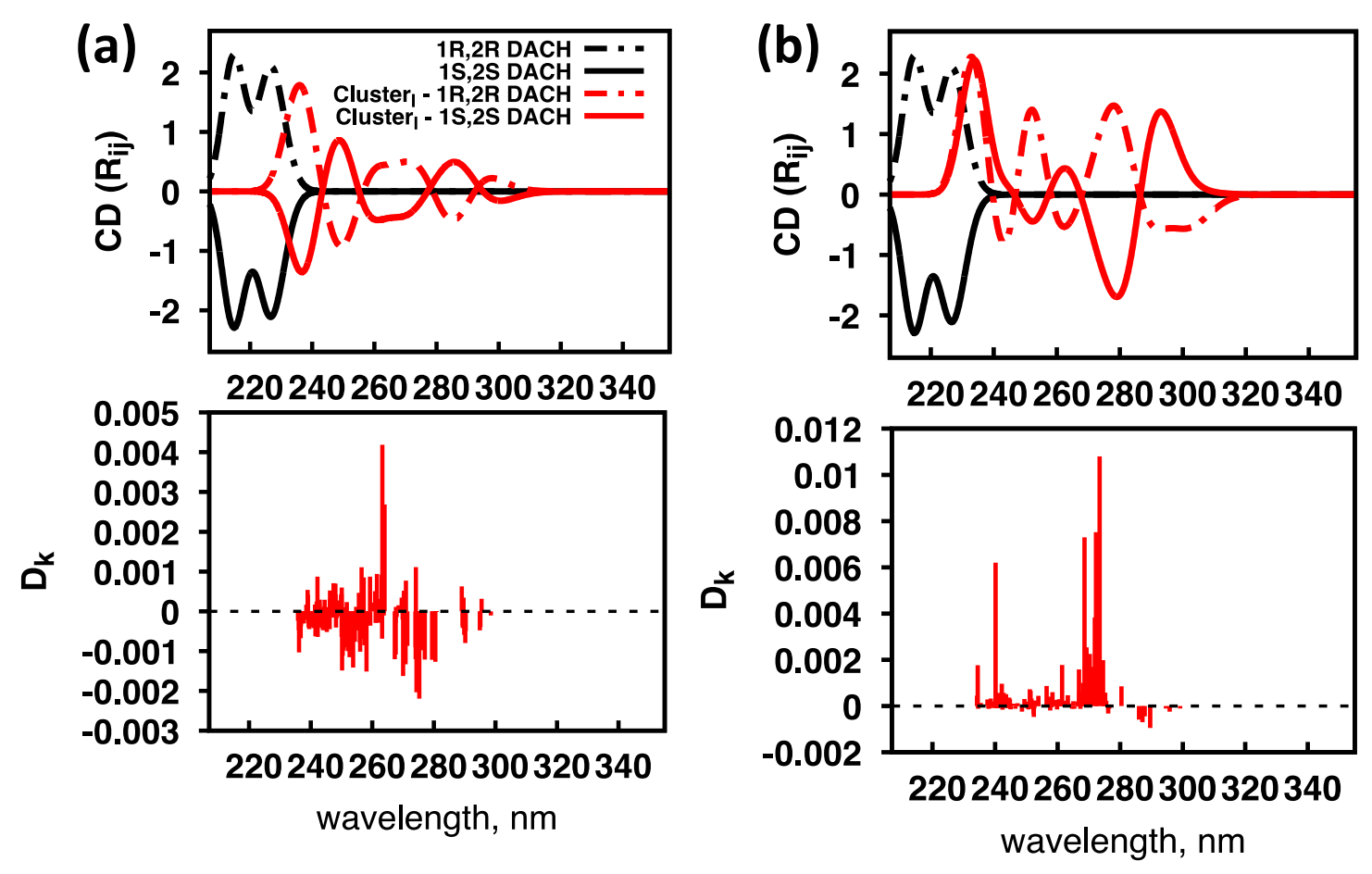

Figure S5: Analysis of charge-transfer excitations for the CsPbI3 cluster with 1S,2S DACH bound to the surface in the (a) monodentate and (b) bridging configuration. Top panels show the joint $C D$ from Figure 2 and the bottom panels show the charge transfer character for each excitation. 

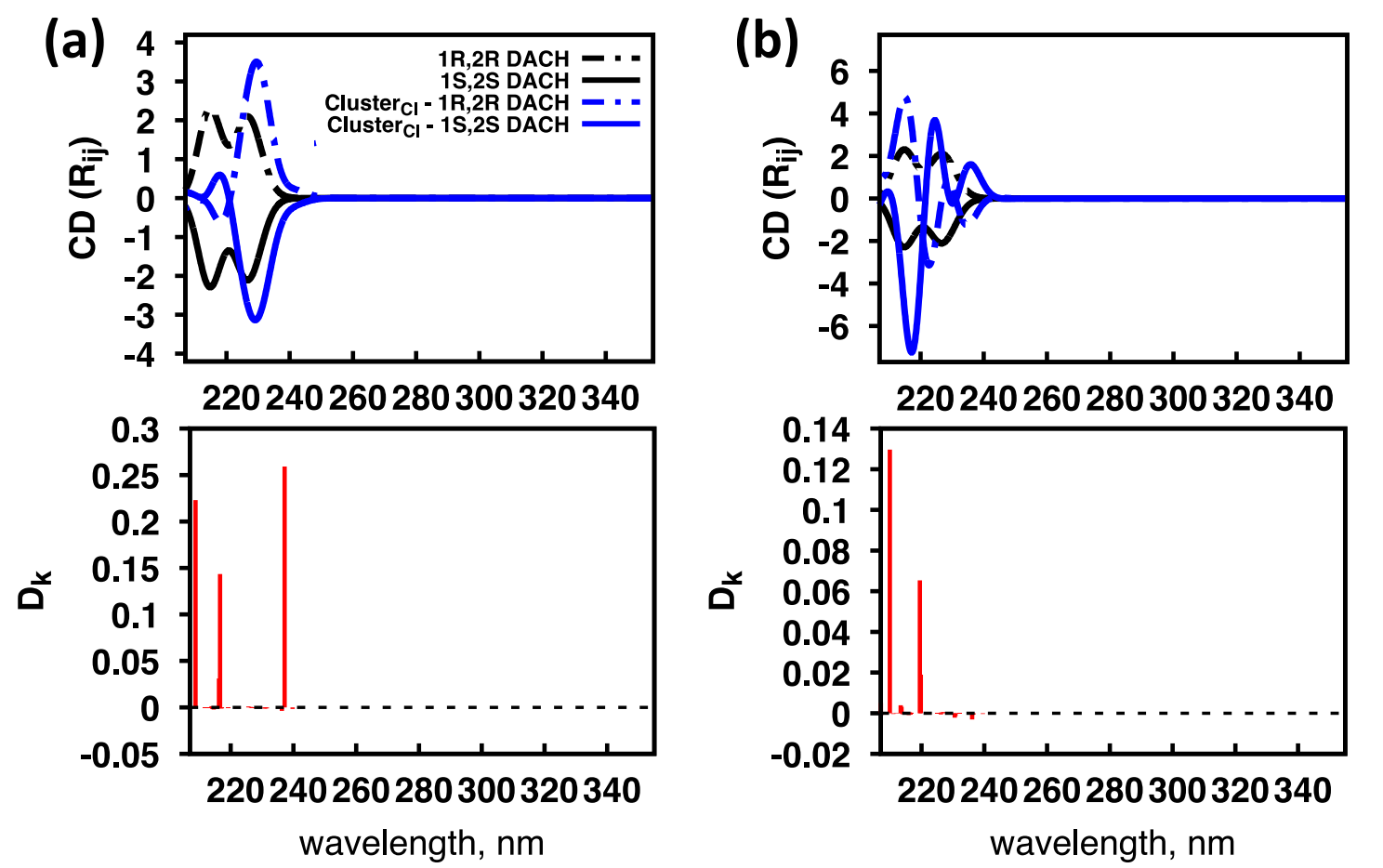

Figure S6: Analysis of charge-transfer excitations for the $\mathrm{CsPbCl}{ }_{3}$ cluster with $1 \mathrm{~S}, 2 \mathrm{~S}$ DACH bound to the surface in the (a) monodentate and (b) bridging configuration. Top panels show the joint $\mathrm{CD}$ spectra from Figure 2 and the bottom panels show the charge transfer character for each excitation.
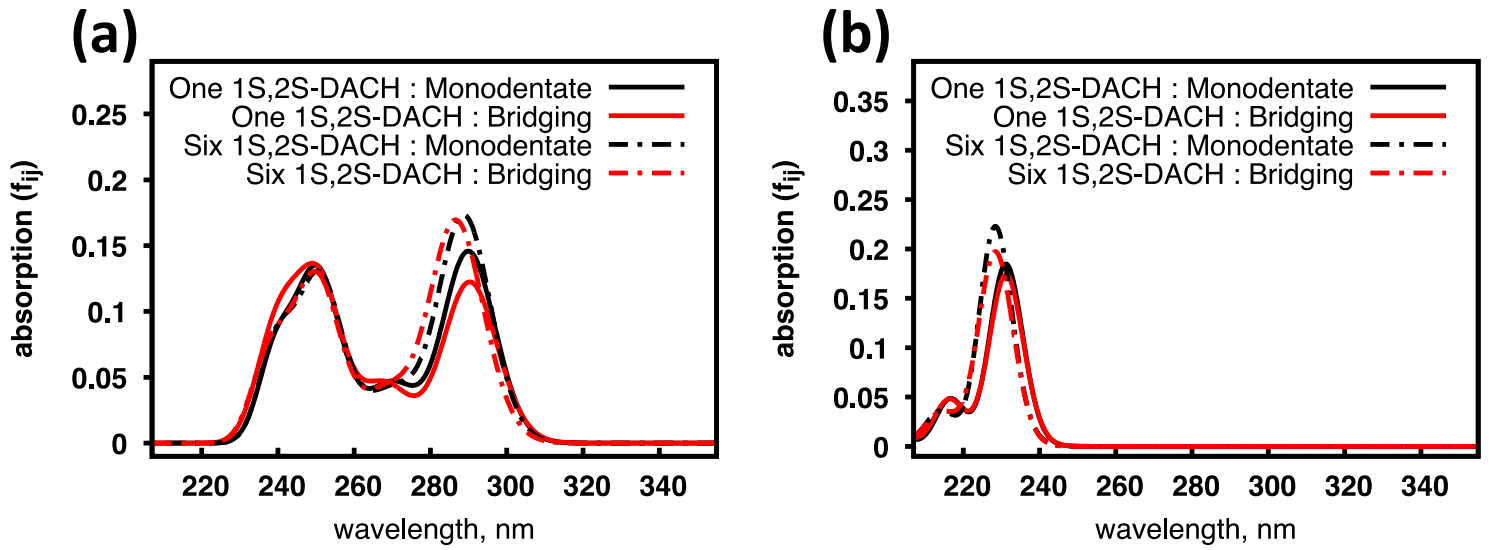

Figure S7: Comparison of computed absorption spectra used to compute the anisotropy factor in Figure 5 for (a) $\mathrm{CsPbl}_{3}$ and (b) $\mathrm{CsPbCl}_{3}$ clusters. The small variation in the absorption spectra verifies that changes in the anisotropy factor are due to the change in CD spectra as a function of surface binding configuration and ligand density. 
(1) Autschbach, J.; Ziegler, T.; van Gisbergen, S. J. A.; Baerends, E. J., Chiroptical properties from time-dependent density functional theory. I. Circular dichroism spectra of organic molecules. J. Chem. Phys. 2002, 116 (16), 6930-6940.

(2) Han, B.; Zhu, Z.; Li, Z.; Zhang, W.; Tang, Z., Conformation Modulated Optical Activity Enhancement in Chiral Cysteine and Au Nanorod Assemblies. JACS 2014, 136 (46), 1610416107.

(3) Gao, X.; Han, B.; Yang, X.; Tang, Z., Perspective of Chiral Colloidal Semiconductor Nanocrystals: Opportunity and Challenge.JACS 2019, 141 (35), 13700-13707.

(4) Jabed, M.; Dandu, N.; Tretiak, S; Kilina, S., Passivating Nucleobases Bring Charge Transfer Character to Optically Active Transitions in Small Silver Nanoclusters. J Phys. Chem. A 2020124 (43), 8931-8942

(5) Frisch, M. J.; Trucks, G. W.; Schlegel, H. B.; Scuseria, G. E.; Robb, M. A.; Cheeseman, J. R.; Scalmani, G.; Barone, V.; Petersson, G. A.; Nakatsuji, H.; Li, X.; Caricato, M.; Marenich, A. V.; Bloino, J.; Janesko, B. G.; Gomperts, R.; Mennucci, B.; Hratchian, H. P.; Ortiz, J. V.; Izmaylov, A. F.; Sonnenberg, J. L.; Williams; Ding, F.; Lipparini, F.; Egidi, F.; Goings, J.; Peng, B.; Petrone, A.; Henderson, T.; Ranasinghe, D.; Zakrzewski, V. G.; Gao, J.; Rega, N.; Zheng, G.; Liang, W.; Hada, M.; Ehara, M.; Toyota, K.; Fukuda, R.; Hasegawa, J.; Ishida, M.; Nakajima, T.; Honda, Y.; Kitao, O.; Nakai, H.; Vreven, T.; Throssell, K.; Montgomery Jr., J. A.; Peralta, J. E.; Ogliaro, F.; Bearpark, M. J.; Heyd, J. J.; Brothers, E. N.; Kudin, K. N.; Staroverov, V. N.; Keith, T. A.; Kobayashi, R.; Normand, J.; Raghavachari, K.; Rendell, A. P.; Burant, J. C.; Iyengar, S. S.; Tomasi, J.; Cossi, M.; Millam, J. M.; Klene, M.; Adamo, C.; Cammi, R.; Ochterski, J. W.; Martin, R. L.; Morokuma, K.; Farkas, O.; Foresman, J. B.; Fox, D. J. Gaussian 16 Rev. C.01, Wallingford, CT, 2016. 